DOI: 10.12957/demetra.2017.22559

\title{
Cuerpo y percepción en una zona rural de México: ¿una preocupación solo de mujeres o también de hombres?
}

\section{Body perception in a rural area of Mexico: is there a concern only women or also men?}

Ana Gabriela Romero Juárez'

Sara Elena Pérez Gil Romo ${ }^{1}$

Sergio Aaron De la Rosa Cruz ${ }^{2}$

1 Instituto Nacional de Ciencias Médicas y Nutrición Salvador Zubirán. Ciudad de México, México.

2 Universidad Autónoma Metropolitana, Unidad Xochimilco, Licenciatura en Nutrición. D.F., México.

Correspondencia / Correspondence Ana Gabriela Romero Juárez

Instituto Nacional de Ciencias Médicas y Nutrición Salvador Zubirán

Ave. Vasco de Quiroga 15, Col. Belisario Domínguez, Sección XVI. Ciudad de México, México.

E-mail:micropollit@gmail.com

\section{Resumen}

Introducción: El presente estudio se inscribe en los trabajos de alimentación y género, entre los cuales se ve al cuerpo como un constructo social. La preocupación por el cuerpo, la imagen corporal y la alimentación se ha convertido actualmente en un problema, sobre todo para las mujeres urbanas. Sin embargo, se sabe poco de lo que sucede entre los hombres y mujeres que viven en las zonas rurales del país. Objetivos: Conocer cómo son, cómo se perciben y cómo desean ser las mujeres y hombres de una comunidad rural y cuáles son algunos de sus saberes alimentarios. Metodología: Se encuestó y midió a 19 hombres y 30 mujeres en una comunidad del Estado de México los que seleccionaron entre 9 figuras, aquellas con las que identificaron su percepción y su deseo. Resultados: Se encontraron algunas diferencias entre los sexos respecto a la percepción corporal: los hombres se perciben más delgados, y algunas mujeres, con sobrepeso. Ambos sexos desean estar más delgados, y se detectaron más alteraciones en la percepción masculina respecto a eso. Los saberes sobre alimentación, cuerpo y salud no mostraron grandes diferencias genéricas. Conclusión: Se resalta la conveniencia de aumentar el número de estudios dirigidos a conocer la percepción y alteración corporal en hombres y mujeres que puedan conducir a prácticas alimentarias de riesgo en ambientes rurales con el propósito de implementar acciones de prevención y tratamiento.

Palabras clave: Cuerpo. Percepción Corporal. Género. 


\section{Abstract}

Introduction: This paper is part of a food and gender research study where the body is seen as a social construct. Concern about the body, body image and food has become a problem, especially for urban women. However, there is little information on what happens to men and women in rural areas of Mexico. Objectives: To investigate how women and men in rural areas perceive their bodies, how they want to look like and what their traditional knowledge about food is. Methodology: In a rural community in the State of Mexico, 19 men and 30 women were surveyed, measured and weighed. They selected 9 pictures, according to their selfperception and their desired figure. Results: There were certain sex differences in body perception: men perceived themselves as thinner and some women perceived themselves as overweight. Both groups wish to be thinner. There is more alteration in male self-perception. There are no gender differences in traditional knowledge about food, body and health. Conclusion: More research is needed on altered body self-perception which may lead to high-risk eating practices in rural environments, so that prevention and treatment can be implemented.

Keywords: Body. Self Perception. Gender.

\section{Introducción}

\section{El problema epidemiológico: algunos datos}

En México, el sobrepeso y la obesidad se han convertido en los problemas más importantes de salud pública. Resultados de la última encuesta nacional sobre salud y nutrición ${ }^{1}$ muestran que la prevalencia combinada de sobrepeso y obesidad (IMC $\geq 25 \mathrm{~kg} / \mathrm{m} 2$ ) es mayor en las mujeres $(73 \%)$ que en los hombres (69\%) y que la prevalencia de obesidad (IMC $\geq 30 \mathrm{~kg} / \mathrm{m} 2$ ) es más alta en el sexo femenino que en el masculino. Al analizar las tendencias de las categorías de índice de masa corporal (IMC) en mujeres de 20 a 49 años de edad, se observa que, en el periodo de 1988 a $2006,,^{2,3}$ la prevalencia de sobrepeso se incrementó a $41,2 \%$ y la de obesidad a $270 \%$. Si bien es cierto que la tendencia de sobrepeso disminuyó 5.1\% entre el año 2006 y 2012, la de obesidad aumentó únicamente $3 \% .^{1,3}$

La prevalencia de obesidad, de acuerdo con otros/as autores/as, también se ha elevado entre las mujeres de los sectores sociales pobres. ${ }^{4-6}$ Estudios llevados a cabo con familias de distintos estratos socioeconómicos en la zona metropolitana de la ciudad de México mostraron, en la población femenina de 18 a 69 años de nivel bajo, una prevalencia de obesidad de $22.5 \%$ en 1995 y $28.7 \%$ 
en 2002., En 2003, se encontró en una población rural pobre una prevalencia combinada de sobrepeso y obesidad de casi $60 \%$ en mujeres y más de $50 \%$ en hombres, con cifras de obesidad de $22.2 \%$ para las primeras y de $13.6 \%$ para los segundos. Las conclusiones de esos estudios giraron en torno a que, desde la década de los noventa, el sobrepeso comenzaba a presentarse con más frecuencia entre las mujeres de los estratos socioeconómicos altos y la obesidad tendía a ser más común en las mujeres de nivel socioeconómico bajo, aunque sin diferencias relevantes entre los diversos estratos y con un patrón similar al de los países desarrollados.

El aumento de otros trastornos del comportamiento alimentario, como la anorexia y la bulimia, y por consiguiente de ciertas prácticas alimentarias de riesgo (no desayunar, dietas de reducción, consumo de productos lights, de los llamados "milagros", de suplementos, entre otros), ha sido detectado en diferentes investigaciones. ${ }^{9,10}$ En un estudio realizado en la Ciudad de México en el que se analizó la información de tres diferentes años, 1997, 2000 y 2003, ${ }^{11,12}$ y cuyo propósito fue identificar las tendencias de ese tipo de prácticas en estudiantes, se observó un incremento en la prevalencia de 1997 a 2003, esto es, para los hombres de $1.3 \%$ a $3.8 \%$, y para las mujeres de $3.4 \%$ a $9.6 \%$. El aumento en la frecuencia de esas conductas en ambos sexos es observado en todas las edades, en particular, entre los varones de 12 y 13 años y las mujeres de 18 y $19 .{ }^{11-13}$

Hasta hace algunos años, se pensaba que la bulimia y la anorexia nerviosa, y por tanto las prácticas alimentarias relacionadas con esos trastornos, las padecían solo personas de nivel socioeconómico elevado, sobre todo de los países industrializados, sin embargo, se las observa cada vez con mayor frecuencia en países en vías de desarrollo y en personas de bajos recursos económicos. $^{14}$

\section{Alimentación, cuerpo e imagen: un nuevo problema}

El estudio del cuerpo se ha convertido en la actualidad en un tema de reflexión en diversas disciplinas. En las ciencias sociales, lo que se denomina teoría social del cuerpo, el enfoque teórico-metodológico, es relativamente nuevo y es el centro de interés en temáticas y diversas especialidades, como la antropología de la salud, de la alimentación y feminista, entre otras. ${ }^{15}$ En las ciencias biológicas, la atención se dirige principalmente hacia el cuerpo como receptor de la ingesta de alimentos y de nutrimentos y su relación con el funcionamiento orgánico.

Son varias las premisas de las que se parte en este texto para comprender y reflexionar acerca de la alimentación y el cuerpo, no desde una visión biológica, sino sociocultural. Comer es una necesidad primaria, y para sobrevivir el ser humano tiene que nutrirse, sin embargo, los alimentos, además de contener nutrimentos y energía, tienen significados. Los seres humanos eligen, jerarquizan y clasifican entre lo que tienen disponible, ${ }^{16-18} \mathrm{y}$, en esos momentos, los aspectos socioculturales del cuerpo y los alimentos relacionados con los valores materiales y simbólicos 
que se otorgan al hecho de comer y no comer cobran relevancia, cuando lo que se pretende es entender qué es el cuerpo desde otra perspectiva diferente a la biomédica.

Comer demasiado, comer poco o no comer nada suele revestirse de múltiples significados sociales y psicológicos más allá de lo nutricional, así como el peso corporal y las formas corporales que de eso se derivan pueden expresar tanto una información acerca de los hábitos alimentarios como de la personalidad, las intenciones e incluso el estatus social de las personas. ${ }^{16,19,20}$

Por lo que respecta a la imagen corporal, existen varias definiciones, pero en este trabajo se retoma la expresada por Raich, ${ }^{21}$ es decir, la imagen como un constructo psicológico complejo, que además de incluir al cuerpo, toma en cuenta las creencias, emociones, pensamientos y conductas asociadas. En otras palabras, la imagen corporal está influenciada por factores históricos, culturales, sociales, individuales y biológicos que varían con el tiempo y abarca múltiples dimensiones, destacándose entre ellas las de género, identidad, estima propia, entre otras. ${ }^{22}$ En particular, la perspectiva de género es una herramienta fundamental que ayuda comprender la construcción identitaria de los hombres y mujeres. El género como proceso de configuración de prácticas sociales involucra directamente al cuerpo, y eso no implica que los hechos biológicos determinen las experiencias sociales de hombres y mujeres, ${ }^{23}$ sino que "el género existe precisamente en la medida en que la biología no determina lo social".

En las últimas décadas, ser delgado se ha convertido en uno de los objetivos principales de la población de las sociedades desarrolladas, una meta impuesta por nuevos modelos de vida según los cuales la imagen corporal parece ser el único sinónimo válido de éxito, felicidad e, incluso, salud. ${ }^{14,24}$ Ser una persona con obesidad constituye, en el momento actual, un auténtico estigma social, y es evidente que la presión social contra el sobrepeso y obesidad se ejerce en forma mucho más acentuada sobre las mujeres, que experimentan de modo especial la presión a favor a la delgadez, manifestado en un desmesurado énfasis puesto sobre el cuerpo, la talla y la forma.,14,16,19,25

Diversos autores y autoras enfatizan que las alteraciones en la imagen corporal han sido consideradas por mucho tiempo como un problema principal y a veces exclusivo de mujeres y que probablemente ésta ha sido la causa de porqué se ha estudiado menos o se diagnostican con menor frecuencia los problemas con relación a la imagen corporal en hombres. Sin embargo, la insatisfacción corporal de los hombres ha aumentado dramáticamente durante las últimas tres décadas, de un $15 \%$ a un $43 \%$, haciendo esas tasas casi comparables a aquellas encontradas en mujeres. ${ }^{24,26,27}$ Los disturbios en la imagen corporal entre los hombres son más complejos que los que se observa es entre las mujeres, ${ }^{28}$ pues mientras que la mayoría de ellas quiere ser más delgadas, los hombres quieren lo mismo, pero a la vez más anchos de lo que son, un patrón que se ha notado entre niños de varias edades y hombres adultos. Esa situación afecta igualmente a aquellos hombres que participan de actividades deportivas ya sea por asuntos de ejercicio o por mantener una imagen corporal particular. 
Fischler ${ }^{29}$ argumenta que un análisis histórico de los modelos corporales mostraría que siempre ha existido una profunda ambivalencia de las representaciones de la gordura y la delgadez y que éstas también han influido de diferentes maneras en los comportamientos alimentarios. A lo largo de las últimas cuatro décadas, se ha presentado una serie de cambios en relación con el ideal de cuerpo, tanto femenino como masculino, de tal manera que el deseo de salud, juventud y atractivo sexual es una poderosa motivación contra la obesidad..$^{11,15,25,30-33}$

\section{El cuerpo de la población que vive en las zonas rurales: cuna nueva preocupación?}

Estudios realizados con mujeres del medio rural de México han aportado algunos datos al tema de la percepción corporal, ${ }^{34-36}$ y las conclusiones, aunque no son definitivas, muestran diferencias según la región y la situación sociocultural y económica de las distintas comunidades, además de la edad, que se repite como un factor determinante en el incremento de la obesidad. Se detecta una subestimación en la percepción corporal entre las mujeres con normopeso, sobrepeso y obesidad, y una sobreestimación en las delgadas, es decir, se observa una alteración en la imagen corporal y una preocupación por la estética.

Ahora bien, datos derivados de estudios con hombres que habitan en las zonas rurales mexicanas son escasos. Keijzer y Rodriguez ${ }^{37}$ comentan que, en el contexto rural, la transición de niño a adulto está marcada por el trabajo más que por el desarrollo biológico, como es el caso de las mujeres. El "amalgamamiento" del cuerpo según la profesión u ocupación demuestra la centralización del trabajo en la construcción de la identidad masculina. El tipo de trabajo y el desgaste son valores agregados en la percepción corporal, y el mayor alcance de los medios de comunicación y materiales audiovisuales importados, muchos de ellos por migrantes que regresan a su lugar de origen (como revistas, películas, videos pornográficos, entre otros), han modificado la percepción corporal de ellos mismos y de las mujeres, así como las expectativas de trabajo. ${ }^{37-40}$ En suma, de acuerdo con los autores revisados, el cuerpo es vivido como un instrumento para cumplir el rol histórico de proveedores de la familia, y la construcción de la identidad masculina se centra en el trabajo, aunque también coexista el deseo de tener un cuerpo atlético, por lo que se recurre a ejercicios, dietas y al uso de suplementos para resaltar la belleza. ${ }^{39,41}$

Con base en lo expuesto anteriormente, la investigación que aquí se presenta es una primera aproximación diferenciadora de la realidad de hombres y mujeres que viven en una zona rural de México acerca de cómo ven su cuerpo, cuyo objetivo central fue conocer cómo son, cómo se perciben y cómo desean ser esas mujeres y hombres. Las interrogantes planteadas fueron: ¿cómo perciben las mujeres y los hombres de las comunidades rurales su cuerpo y cuáles son sus principales representaciones?, ¿hay diferencias de género en torno a cómo son, cómo se perciben y cómo quisieran ser?, cexisten ideales de cuerpo entre las mujeres y hombres de esas zonas, y 
de ser así, cuáles son? y chay diferencias entre los saberes de hombres y mujeres acerca de la alimentación y la salud?

\section{La comunidad de San Martín Cachihuapan}

El presente estudio se realizó en la comunidad San Martín Cachihuapan, uno de los pueblos más antiguos del municipio de Villa del Carbón, en el Estado de México. La población total, de acuerdo al último censo, era de 1532 personas, de las cuales 751 eran hombres y 781, mujeres. En esa región, incluso cuando se asentó un grupo de indígenas otomíes provenientes del norte del país, en la actualidad, la población ya no habla lenguas indígenas. Para 2010, se había registrado un alto grado de marginación en la población, 6.2\% de las viviendas tenían piso de tierra, 18.8\% no contaban con drenaje, $3.4 \%$ no tenían luz eléctrica y $9.4 \%$ no disponían de agua entubada. El 27.4\% de la población no era derechohabiente de servicios de salud, y el 8.9\% de la población mayor de 15 años estaba registrada como analfabeta. Las principales actividades económicas son la agricultura, la ganadería y la explotación forestal, esta última debido a que la comunidad se encuentra ubicada en una zona boscosa de clima templado. ${ }^{42}$

\section{Metodología}

La información que aquí se presenta es producto de una investigación observacional, transversal, descriptiva y de tipo cuali-cuantitativo realizada en 2015 en la comunidad de San Martín Cachihuapan, en el Estado de México. Como ya se mencionó anteriormente, este trabajo constituye un primer abordaje de la percepción corporal masculina en una zona rural. Una primera diferencia genérica encontrada fue el hecho de que varios hombres no aceptaron participar en el estudio, pues el tema de la alimentación y del cuerpo, según sus comentarios, es únicamente de mujeres. Fue así que se encuestó solo a 19 hombres y a 30 mujeres, que tuvieran entre 15 y 65 años de edad.

El instrumento de recolección de la información fue un cuestionario conformado por preguntas abiertas y cerradas y validado previamente en poblaciones rurales del estado de Oaxaca. La aplicación del instrumento de recolección de la información se dio en los hogares de cada una de las personas que aceptaron participar en el estudio, y la toma de medidas antropométricas, peso y estatura se llevó a cabo en el centro de salud.

Para cumplir con los objetivos de la investigación y dar respuesta a las interrogantes, se incluyen en este trabajo solo datos relacionados con la ocupación, así como el Índice de Masa Corporal (IMC) registrado (cómo son), la autopercepción de la imagen corporal (cómo se ven) y el anhelo a 
cierto tipo de cuerpo (cómo el encuestado desearía ser). La imagen corporal percibida y la imagen anhelada se obtuvieron solicitando a las mujeres y hombres que seleccionaran entre nueve figuras de la escala del Standard Figural Stimuli (SFS) que van desde la desnutrición severa (tipo 2) hasta la obesidad 3, aquélla que, según su percepción, correspondía a su imagen corporal actual y a la que les gustaría tener (Figura 1). ${ }^{43}$

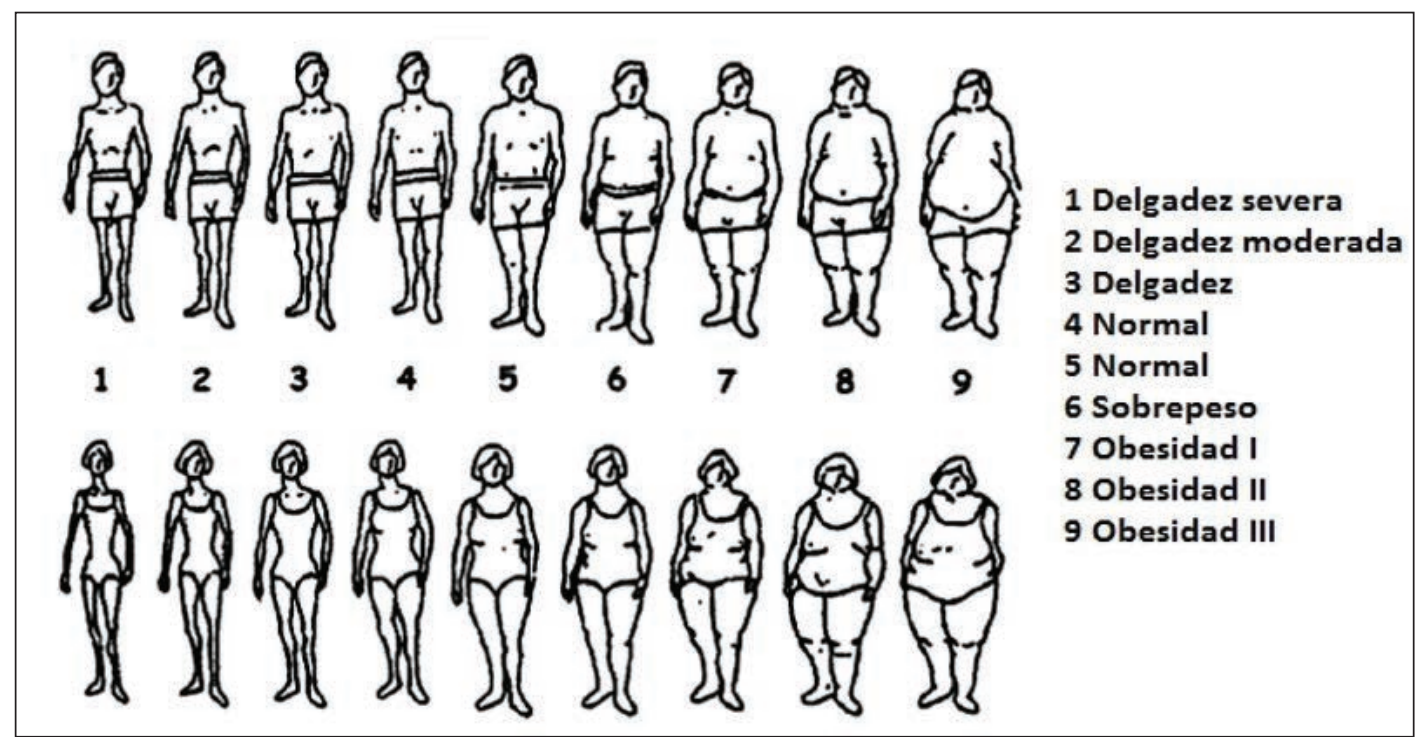

Figura 1. Standard Figura 1 Stimuli (SFS)

El análisis de los datos derivados de la composición y la percepción corporal se realizó con el auxilio del paquete estadístico SPSS versión 21, en el cuál se aplicó la prueba de tablas de contingencia para cruzar las variables cómo soy y cómo me percibo, cómo soy y cómo deseo ser, y así conocer la coincidencia, sobreestimación y subestimación y, por último, cómo me percibo y como deseo ser, para identificar una posible insatisfacción corporal.

Finalmente, por lo que se refiere a la información relacionada con algunos saberes alimentarios, producto de las preguntas abiertas, se presentan pequeñas narraciones agrupadas en función de las categorías seleccionadas. 


\section{Resultados Y Discusión}

\section{Cómo son los hombres y mujeres, cómo se perciben y cómo desean ser}

El promedio de edad de los19 hombres fue 36 años, y el de las 30 mujeres, 38 años. La mayor parte de los varones comentó emplearse como choferes de vehículos del transporte público, otros como obreros, y solo dos de ellos se dedicaban a las actividades agrícolas. Las mujeres manifestaron dedicarse principalmente a las actividades del hogar, algunas trabajaban como empleadas domésticas y otras se dedicaban al comercio. En ambos sexos, se detectaron estudiantes (tres hombres y cuatro mujeres).

Por lo que se refiere a su composición corporal, el promedio del peso entre los varones fue de $77 \mathrm{Kg}$, con un mínimo de $59 \mathrm{Kg}$ y un máximo de $103 \mathrm{Kg}$, y entre las mujeres fue de $64 \mathrm{Kg}$, con un rango entre 48 y $80 \mathrm{Kg}$. El promedio para la estatura de los hombres fue de $1.68 \mathrm{~m}$, variando de 1.58 a 1.80, y de las entrevistadas fue de $1.55 \mathrm{~m}$, con un mínimo de 1.40 y un máximol.68. En cuanto al estado nutricional, evaluado a través del IMC, el promedio fue de 27.2 entre los hombres y 26.7 entre las mujeres, con una variación de 21.1 a 35.2 para los primeros y de 21.0 a 34.2 para las segundas, es decir, la diferencia en la situación nutricional entre los sexos, al utilizar el IMC, fue mínima. Tanto para hombres como para mujeres, el promedio del IMC se ubicó en sobrepeso y los rangos máximos (34.2 y 35.2) dan cuenta de obesidad de segundo grado.

En la Tabla 1, en la primera columna, "cómo soy" (estado nutricional de los hombres y mujeres medido a través del IMC), se observan datos muy parecidos en los dos sexos: no se registró bajo peso y la diferencia en la normalidad fue de solo 5 puntos y en el sobrepeso de seis puntos, esto es, aunque más mujeres presentaran normopeso y más hombres sobrepeso, las diferencias porcentuales fueron muy pequeñas; y por lo que respecta a la obesidad, la prevalencia fue igual en ambos sexos. Es importante resaltar que, aún cuando no se encontraron grandes diferencias entre los hombres y las mujeres, los datos disponibles muestran un problema de sobrepeso y obesidad en la población estudiada, ya que la prevalencia de esos padecimientos resultó por arriba del 60\%, situación que no difiere a lo reportado en las últimas encuestas nacionales sobre alimentación y nutrición realizadas en el país. ${ }^{1}$ En el momento actual, México, junto con Estados Unidos de América ocupan los primeros lugares de prevalencia mundial de sobrepeso y obesidad en la población adulta.

Con el propósito de conocer las diferencias entre "cómo soy" y "cómo me veo", en la misma Tabla, se destaca en la segunda columna que los hombres se percibieron más delgados y más normales de lo que se registró, y aquellos que fueron clasificados con obesidad no pensaban sufrir ese padecimiento. Con las mujeres, sucedió algo similar (Tabla 1), sin embargo, en la autopercepción femenina, "cómo me veo", aún cuando algunas mujeres seleccionaron las figuras delgadas y con normalidad, un poco más de la tercera parte eligió la silueta con sobrepeso, es decir, se percibió 
con kilos de más. El tema de la autopercepción corporal resulta de interés, ya que es justo aquí donde radica una de las principales diferencias entre hombres y mujeres: más mujeres percibieron que su peso era excesivo.

En la tercera columna, "cómo deseo ser", se observa que tanto a hombres como a mujeres no les agradaría tener un cuerpo con sobrepeso y obesidad; solo una mujer eligió una figura con sobrepeso, lo cual llama la atención. El deseo corporal se distribuyó de manera similar en los hombres entre el bajo peso y la normalidad, y en las mujeres, para los mismos rubros, sí hubo diferencias: una tercera parte optó por bajo peso y casi dos terceras partes, por normalidad. Aunque en menor cantidad, podría afirmarse que la figura con normalidad fue la más elegida por los hombres. De lo anterior, se desprende que, al igual que los hombres, las mujeres también desean ser más delgadas de lo que son, ya que los porcentajes de sobrepeso y obesidad disminuyeron entre "cómo soy" y "cómo me veo" y aumentaron los de normalidad y bajo peso. (Tabla 1). El deseo por un cuerpo más delgado se hizo evidente en ambos sexos, lo que, en la población de San Martín, al parecer no muestra una diferencia atribuible al género.

Tabla 1. "Cómo soy, cómo me veo y cómo deseo ser", según un grupo de hombres y mujeres de San Martín Cachihuapan, México.

\begin{tabular}{|l|c|c|c|c|c|c|}
\hline \multirow{2}{*}{} & \multicolumn{2}{|c|}{ Cómo soy } & \multicolumn{2}{c|}{ Cómo me veo } & \multicolumn{2}{c|}{ Cómo deseo ser } \\
\cline { 2 - 8 } & $\mathrm{N}$ & $\%$ & $\mathrm{~N}$ & $\%$ & $\mathrm{~N}$ & $\%$ \\
\hline Bajo peso & 0 & 0 & 10 & 52.7 & 9 & 47.4 \\
\hline Normal & 6 & 31.6 & 7 & 36.8 & 10 & 52.6 \\
\hline Sobrepeso & 10 & 52.6 & 2 & 10.5 & 0 & 0 \\
\hline Obesidad & 3 & 16.8 & 0 & 0 & 0 & 0 \\
\hline Total & 19 & 100.0 & 19 & 100.0 & 19 & 100.0 \\
\hline & & & Mujeres & & & 33.3 \\
\hline Bajo peso & 0 & 0 & 5 & 16.7 & 10 & 63.4 \\
\hline Normal & 11 & 36.7 & 13 & 43.3 & 19 & 3.3 \\
\hline Sobrepeso & 14 & 46.7 & 11 & 36.7 & 1 & 0 \\
\hline Obesidad & 5 & 16.7 & 1 & 3.3 & 0 & 100.0 \\
\hline Total & 30 & 100.0 & 30 & 100.0 & 30 & \\
\hline
\end{tabular}


La coincidencia, sobreestimación y subestimación del cuerpo de los hombres y mujeres se presenta en las tablas 2, "cómo soy y cómo me percibo", 3, "cómo soy y cómo desearía ser" y 4, "cómo me percibo y cómo desearía ser", que da cuenta de una insatisfacción. Así pues, al comparar "cómo son" los hombres con el cuerpo percibido, en la Tabla 2, se aprecia que de los seis hombres clasificados dentro de la normalidad ninguno coincidó su clasificación nutricia con su percepción, ya que todos se subestimaron al elegir la figura con bajo peso, a diferencia de las 11 mujeres, que, habiendo sido calificadas con normopeso, solo la mitad de ellas fue coincidente, es decir, se identificó con la figura a la que pertenece; y de las restantes, tres se subestimaron y dos se sobreestimaron al elegir figuras que no correspondían a su IMC (ver Tabla 2).

Tabla 2. Relación entre "cómo soy y cómo me veo" en un grupo de hombres y mujeres de San Martín Cachihuapan, México.

\begin{tabular}{|c|c|c|c|}
\hline \multicolumn{4}{|c|}{ Con un IMC normal } \\
\hline \multicolumn{2}{|r|}{6 hombres } & \multicolumn{2}{|r|}{11 mujeres } \\
\hline $\begin{array}{l}\text { Ninguno } \\
\text { coincidió } \\
\text { en su } \\
\text { percepción }\end{array}$ & $\begin{array}{l}6(100 \%) \text { se identificaron } \\
\text { con bajo peso (se } \\
\text { subestimaron) }\end{array}$ & $\begin{array}{l}6(54.4 \%) \\
\text { Sí } \\
\text { coincidieron }\end{array}$ & $\begin{array}{l}3(27.3 \%) \text { se identificaron con } \\
\text { bajo peso (se subestimaron) } \\
2(18.2 \%) \text { se identificaron } \\
\text { con sobrepeso (se } \\
\text { sobrestimaron) }\end{array}$ \\
\hline \multicolumn{4}{|c|}{ Con sobrepeso } \\
\hline \multicolumn{2}{|r|}{8 hombres } & \multicolumn{2}{|r|}{14 mujeres } \\
\hline $\begin{array}{l}\text { Ninguno } \\
\text { coincidió } \\
\text { en su } \\
\text { percepción }\end{array}$ & $\begin{array}{l}3(37.5 \%) \text { se identificaron } \\
\text { con bajo peso (se } \\
\text { subestimaron) } \\
5(62.5 \%) \text { como normales } \\
\text { (se subestimaron) }\end{array}$ & $\begin{array}{l}6(42.9 \%) \\
\text { Sí } \\
\text { coincidieron }\end{array}$ & $\begin{array}{l}2(14.3 \%) \text { se identificaron con } \\
\text { bajo peso (se subestimaron) } \\
5(35.7 \%) \text { como normales (se } \\
\text { subestimaron) } \\
1(7.1 \%) \text { con obesidad (se } \\
\text { sobrestimó) }\end{array}$ \\
\hline \multicolumn{4}{|c|}{ Con obesidad } \\
\hline \multicolumn{2}{|r|}{5 hombres } & \multicolumn{2}{|r|}{5 mujeres } \\
\hline $\begin{array}{l}\text { Ninguno } \\
\text { coincidió } \\
\text { en su } \\
\text { percepción }\end{array}$ & $\begin{array}{l}1 \text { (20\%) se identificó con } \\
\text { bajo peso (se subestimó) } \\
2(40 \%) \text { como normales } \\
\text { (se subestimaron) } \\
2(40 \%) \text { con sobrepeso (se } \\
\text { subestimaron) }\end{array}$ & $\begin{array}{l}\text { Ninguna } \\
\text { coincidió } \\
\text { en su } \\
\text { percepción }\end{array}$ & $\begin{array}{l}2(40 \%) \text { se identificaron como } \\
\text { normales (se subestimaron) } \\
3(60 \%) \text { con sobrepeso (se } \\
\text { subestimaron) }\end{array}$ \\
\hline
\end{tabular}


De los ocho hombres clasificados con sobrepeso, ninguno coincidió con su autopercepción, pues tres se identificaron con bajo peso y cinco con normalidad, mientras que, en el caso de la población femenina con sobrepeso, la elección de la figura resultó más diversa, ya que en seis de las mujeres se encontró coincidencia con su cuerpo, siete se subestimaron y seleccionaron un modelo anatómico delgado o normal y una se sobreestimó. Por último, de los cinco varones y cinco mujeres diagnosticados/as con obesidad, el total se subestimó al elegir alguna figura más delgada (ver Tabla 2). En suma, las alteraciones en la percepción de la imagen corporal afectaron a más de la mitad de la población estudiada, siendo más frecuentes en hombres que en mujeres. En el caso de los hombres, esas alteraciones se debieron a una subestimación del peso — la totalidad de la población masculina se percibió con kilos de menos-, mientras que en las mujeres aparecieron algunos casos de sobrestimación.

En cuanto a la relación entre "cómo son" y "cómo desean ser" (Tabla 3), se resalta lo siguiente: de los seis hombres clasificados como normales, cuatro de ellos manifestaron desear el cuerpo con normalidad, esto es, coincidieron, y los dos restantes sí desearon un cuerpo con bajo peso. Y de las mujeres, de las 11 clasificadas como normales, ocho no anhelan un cuerpo diferente, se sienten conformes como están y tres manifestaron desear una figura con bajo peso. Por lo que respecta a los ocho hombres que tuvieron sobrepeso, ninguno expresó estar a gusto con su cuerpo, pues cinco eligieron una figura con bajo peso y tres la silueta considerada como normal, en tanto que las mujeres solo una está conforme y en el resto, la inconformidad la manifestaron eligiendo la figura calificada como normal o con bajo peso. Al igual que lo encontrado con la relación entre "cómo son" y "cómo me veo", al interrogar a los que presentaron obesidad, cinco hombres y cinco mujeres, ninguno/a coincidió su situación nutricional con su deseo, pues los diez seleccionaron figuras normales y de bajo peso (ver Tabla 3). En otras palabras, alrededor del $70 \%$ de los hombres y de las mujeres anhelan un cuerpo más esbelto, no necesariamente con delgadez extrema. La preocupación por el cuerpo ya no es una característica solo de mujeres urbanas, pues las sobrevaloraciones o subvaloraciones de la forma y tamaño del cuerpo y la preocupación que eso conlleva también comienzan a presentarse en mujeres y hombres que viven en zonas rurales pobres del país, como se aprecia en este trabajo. 
Tabla 3. Relación entre "cómo soy y como deseo ser" en un grupo de hombres y mujeres de San Martín Cachihuapan, México.

\begin{tabular}{|c|c|c|c|}
\hline \multicolumn{4}{|c|}{ Con IMC Normal } \\
\hline \multicolumn{2}{|r|}{6 hombres } & \multicolumn{2}{|r|}{11 mujeres } \\
\hline $\begin{array}{l}4(66.7 \%) \\
\text { Coincidieron } \\
\text { con su deseo }\end{array}$ & $\begin{array}{l}2(33.3 \%) \text { desearon una } \\
\text { figura con bajo peso }\end{array}$ & $\begin{array}{l}8(72.7 \%) \text { Sí } \\
\text { coincidieron } \\
\text { con su deseo }\end{array}$ & $\begin{array}{l}3(27.3 \%) \text { desearon una } \\
\text { figura con bajo peso }\end{array}$ \\
\hline \multicolumn{4}{|c|}{ Con sobrepeso } \\
\hline \multicolumn{2}{|r|}{8 hombres } & \multicolumn{2}{|r|}{14 mujeres } \\
\hline $\begin{array}{l}\text { Ninguno } \\
\text { coincidió } \\
\text { con su deseo }\end{array}$ & $\begin{array}{l}5(62.5 \%) \text { desearon una } \\
\text { figura con bajo peso } \\
3(37.5 \%) \text { desearon una } \\
\text { figura o normal }\end{array}$ & $\begin{array}{l}1(7.1 \%) \text { Sí } \\
\text { coincidió con } \\
\text { su deseo }\end{array}$ & $\begin{array}{l}5(35.7 \%) \text { desearon una } \\
\text { figura con bajo peso } \\
8(57.1 \%) \text { desearon una } \\
\text { figura normal }\end{array}$ \\
\hline \multicolumn{4}{|c|}{ Con obesidad } \\
\hline \multicolumn{2}{|r|}{5 hombres } & \multicolumn{2}{|r|}{5 mujeres } \\
\hline $\begin{array}{l}\text { Ninguno } \\
\text { coincidió } \\
\text { con su deseo }\end{array}$ & $\begin{array}{l}2(40 \% \%) \text { desearon una } \\
\text { figura con bajo peso } \\
3(60 \%) \text { desearon una } \\
\text { figura normal }\end{array}$ & $\begin{array}{l}\text { Ninguna } \\
\text { coincidió con } \\
\text { su deseo }\end{array}$ & $\begin{array}{l}2(40 \%) \text { desearon una } \\
\text { figura con bajo peso } \\
3(60 \%) \text { desearon una } \\
\text { figura o normal }\end{array}$ \\
\hline
\end{tabular}

Debido a que la disparidad entre la percepción y el deseo de los individuos genera distorsión, discrepancia e insatisfacción con su cuerpo, y además es lo que los hace vulnerables a padecer algún trastorno de la conducta alimentaria, se cruzó la información de "cómo se perciben y cómo desearían ser". En la Tabla 4, se observa que 12 hombres y 16 mujeres presentaron discrepancias entre su percepción y su deseo, lo que significa, en términos porcentuales, que el $63 \%$ de los primeros y el $53 \%$ de las segundas se encuentran insatisfechos con su cuerpo. En ese cuadro, llama la atención el número tan elevado de hombres que manifiestan una distorsión e insatisfacción corporal. 
Tabla 4. Relación entre "cómo me veo y cómo deseo ser" en un grupo de hombres y mujeres de San Martín Cachihuapan, México.

\begin{tabular}{|c|c|c|c|}
\hline \multicolumn{4}{|c|}{ Que se percibieron con una figura normal } \\
\hline \multicolumn{2}{|r|}{7 hombres } & \multicolumn{2}{|r|}{13 mujeres } \\
\hline $\begin{array}{l}3(43 \%) \\
\text { coincidieron }\end{array}$ & $\begin{array}{l}4(57 \%) \text { eligieron la figura } \\
\text { con bajo peso }\end{array}$ & $\begin{array}{l}9(69 \%) \\
\text { coincidieron }\end{array}$ & $\begin{array}{l}3(23 \%) \text { eligieron la figura } \\
\text { con bajo peso } \\
1(8 \%) \text { eligió la figura con } \\
\text { sobrepeso }\end{array}$ \\
\hline \multicolumn{4}{|c|}{ Que se percibieron con bajo peso } \\
\hline \multicolumn{2}{|r|}{10 hombres } & \multicolumn{2}{|r|}{5 mujeres } \\
\hline $\begin{array}{l}4(40 \%) \\
\text { coincidieron }\end{array}$ & $\begin{array}{l}6(60 \%) \text { eligieron la figura } \\
\text { con normalidad }\end{array}$ & $\begin{array}{l}5(100 \%) \\
\text { coincidieron }\end{array}$ & \\
\hline \multicolumn{4}{|c|}{ Que se percibieron con sobrepeso } \\
\hline \multicolumn{2}{|r|}{2 hombres } & \multicolumn{2}{|r|}{11 mujeres } \\
\hline $\begin{array}{l}\text { Ninguno } \\
\text { coincidió }\end{array}$ & $\begin{array}{l}1(50 \%) \text { eligió la figura con } \\
\text { bajo peso } \\
1 \text { ( } 50 \% \text { eligió la figura con } \\
\text { normalidad }\end{array}$ & $\begin{array}{l}\text { Ninguna } \\
\text { coincidió }\end{array}$ & $\begin{array}{l}2(18 \%) \text { eligieron la figura } \\
\text { con bajo peso } \\
9(82 \%) \text { eligieron la figura } \\
\text { con normalidad }\end{array}$ \\
\hline \multicolumn{4}{|c|}{ Que se percibieron con obesidad } \\
\hline \multicolumn{2}{|r|}{ ninguno } & \multicolumn{2}{|r|}{1 mujer } \\
\hline & & No coincidió & $\begin{array}{l}1(100 \%) \text { eligió la figura } \\
\text { con normalidad }\end{array}$ \\
\hline
\end{tabular}

\section{Algunos saberes sobre el cuerpo y la alimentación}

Ahora bien, con el propósito de conocer si las y los entrevistados relacionan la salud con lo que comen, se realizaron varias preguntas. Una de ellas fue para identificar cuál era su sentimiento hacia su cuerpo, si se sentían conformes con él o no. Se encontró que más del $80 \%$ de los hombres se sienten "a gusto con su cuerpo", a diferencia de las mujeres, de las cuales solo el $63 \%$ manifestó conformidad. 
No se detectaron grandes diferencias en las razones aducidas por los hombres y las mujeres acerca de su satisfacción corporal, la resignación fue un elemento importante como se aprecia en las siguientes expresiones, "pues ya soy asî", "así nací", "me acepto como soy". Cabe resaltar que solo en el caso de las mujeres la insatisfacción se relacionó con los aspectos estéticos, por ejemplo, "me veo muy gorda", "así pues estoy fea", "no me gusta cómo me veo". La gordura es un estigma en particular para las mujeres. Para los hombres estar gordo representa una alteración o impedimento sustancial de las funciones como se aprecia en lo siguiente, "pues porque tengo dolencias" y "es que es una limitante para hacer cosas y trabajar".

Al cuestionar acerca de si los alimentos se relacionan con la salud, las respuestas fueron afirmativas en ambos sexos, y las razones que más se mencionaron fueron, entre los hombres: "los alimentos dan energía y vitalidad", "los alimentos te previenen de enfermedades", "comer bien es igual a estar bien y tener salud"; y las mujeres hicieron hincapié en que "los alimentos influyen en la salud", "consumir mucho engorda" y "comer poco adelgaza".

Para conocer un poco más este tema, se les preguntó acerca de quiénes eran más sanos, si las personas gordas o las flacas, y los resultados mostraron que la mayoría de los encuestados de ambos sexos, $67 \%$ de los hombres y $77 \%$ de las mujeres, piensan que los delgados son más saludables; el $16 \%$ de ambos grupos expresaron que tanto gordos como flacos "se enferman igual". No obstante, mientras que el 13\% de los hombres considera a los gordos como más saludables, solo el 3\% de las mujeres comparte esa opinión, el resto respondió no saber. Entre los argumentos expresados por la población masculina para justificar por qué las personas obesas tienen más salud, se resaltan: "porque comen lo que tiene que comer", "porque se alimentan bien", "por herencia", "porque tienen buenos hábitos de alimentación”; en el caso de las mujeres, sin llegar a elogiar la delgadez, aquellas que asocian los cuerpos delgados con cuerpos saludables mencionaron: "porque tienen menos grasa", "se cuidan más, hacen ejercicio y dieta", "se ven mejor"; y solo aquella que consideró a las gordas más sanas argumentó que es debido a que tienen "mayores defensas", es decir, coexisten dos o más argumentos relacionados con este tema. Hasta dónde el discurso médico y los medios de comunicación influyen en estas representaciones sobre la alimentación, la salud y el cuerpo en la población que vive en las zonas rurales del país es un tema a investigar con más profundidad.

Debido a que los seres humanos tienden a clasificar los alimentos y llegan a convertir a algunos de ellos en los principales enemigos del sobrepeso y la obesidad, en la presente investigación, se buscó conocer las posibles diferencias o similitudes entre las respuestas expresadas por los hombres y las mujeres sobre el tema. En términos generales, no hay diferencias, pues así como la gran mayoría de las mujeres encuestadas responsabilizó a los productos industrializados de ser los causantes de la obesidad, los hombres mencionaron que el consumo de ciertos productos con alto contenido de sal, azúcar y calorías engordan más, como muestra el siguiente comentario: "la comida chatarra hace que las personas enfermen y engorden", es decir, los productos industrializados. 
Ambos sexos también calificaron otros alimentos (pan, grasas, tortilla) como engordadores. Solo un pequeño porcentaje de los/las entrevistados/as le otorgó a la carne y al huevo atributos negativos. En cuanto a los alimentos que menos daño causan, para ambos sexos, se encuentran las frutas y verduras, siguiéndoles los cereales y leguminosas y, al final, los productos de origen animal. En suma, no se observaron diferencias atribuibles al rol de género a sus saberes sobre los alimentos, así como tampoco a las enfermedades causadas por una alimentación "inadecuada”, tanto para los hombres como para las mujeres de la comunidad, la diabetes y la presión arterial alta en primer lugar, siguiéndole la anemia, la desnutrición, y posteriormente las enfermedades del estómago y la obesidad "tienen que ver con los alimentos que se consumen". Los saberes sobre la alimentación y el cuerpo no difieren mucho a lo expresado en el discurso médico, en otras palabras, por un lado los hombres y las mujeres asocian el tipo de alimentos que consumen con ciertas enfermedades que pueden llegar a desarrollar como la diabetes, la hipertensión, la anemia y la desnutrición, y por el otro establecen la relación entre la cantidad de alimentos que se consume y la obesidad.

\section{Conclusiones}

Antes que nada, es importante señalar que en ninguna medida los datos que aquí se presentan son representativos de las mujeres y de los hombres que viven en el medio rural mexicano. La información no es resultado de una muestra, sino de individuos de la comunidad de San Martín Cachihuapan, Estado de México, que, como ya fue señalado, aceptaron ser encuestados y medidos. El número de hombres fue menor, debido a su rechazo a contestar un cuestionario sobre alimentación y cuerpo y a ser medidos y pesados. La experiencia vivida en esta investigación en relación con la aceptación a responder el cuestionario mostró una diferencia entre los géneros, ya que, por un lado, la alimentación como parte del rol tradicional de género de las mujeres es un tema que solo les concierne a ellas $^{44} y$, por el otro, los hombres, como lo expresan algunos autores, ${ }^{40,41}$ hablan de "el" cuerpo y no de "mi" cuerpo. Sin embargo, debido a la escasez de datos sobre el cuerpo para los hombres de comunidades rurales, se considera que este es un primer paso para conocer lo que la población masculina que vive en esas zonas percibe y sabe.

La mayoría de los estudios sobre percepción corporal ha sido de tipo cuantitativo, los instrumentos de recolección, diferentes, y los sujetos de estudio han sido escolares preuniversitarios/as, universitarios/as y atletas particularmente de zonas urbanas, lo que hace imposible su comparación con el presente trabajo.

Sobre el tema de los instrumentos de recolección, cabe subrayar la necesidad de elaborar siluetas más afines a la realidad rural de México y se coincide con algunos autores que han criticado las escalas de siluetas, ya que un concepto que es continuo no se debe evaluar con un número limitado de figuras. ${ }^{45}$ En ese sentido, utilizar figuras o modelos anatómicos apegados a 
la realidad de las poblaciones del estudio y continuar con esta temática es crucial para tener un mayor acercamiento a la percepción corporal entre la población adulta, adolescente e infantil del medio rural mexicano, ya que una distorsión de esa imagen puede ser un factor desencadenante en la adopción de ciertas prácticas alimentarias de riesgo. Se tiene información ${ }^{35,36}$ de que hay adolescentes que padecen anorexia en dichas comunidades, de que hay una preocupación por el cuerpo en mujeres adultas y de que se consumen algunos productos lights, como refrescos, yogures, leche y mayonesa, así como otros considerados como "milagrosos para no engordar". Asimismo, con el pretexto de disminuir la obesidad, varios programas alimentarios tienen entre sus componentes la práctica de ejercicio físico, particularmente para las mujeres, para que se continúe siendo un/a beneficiario/a. No se desestima la prevalencia de obesidad en los hombres, no obstante, ese padecimiento es más estigmatizado en las mujeres.

Después de haber mencionado algunas de las limitaciones del estudio y retomado los resultados con base en los objetivos planteados, se puede concluir lo siguiente: la percepción del cuerpo da cuenta de la presencia de una diferencia de sexo, en tanto que los hombres encuestados en San Martín se percibieron más delgados de lo que son, a diferencia de las mujeres que se percibieron más gordas, sin embargo, no se puede afirmar que eso es una cuestión de género, pues existe la posibilidad de que los participantes, como ha ocurrido en estudios anteriores con mujeres de algunas zonas rurales, frente al hecho de tener que elegir una figura, dudaran y no eligieron la silueta más próxima a su percepción. Lo anterior está documentado para el caso de mujeres que mostraron asombro y duda en el momento de tener que elegir una de las figuras.

El deseo por un cuerpo más delgado se hizo evidente en ambos sexos, lo que no muestra una diferencia clara atribuible al género, así como las alteraciones en la percepción de la imagen corporal no revelaron grandes diferencias; eso se presentó en más de la mitad de la población estudiada de ambos sexos, siendo más frecuente en hombres que en mujeres. En el caso de los primeros, las alteraciones se debieron a una subestimación del peso y, en el de las mujeres, se registró una sobrestimación. Estudios cuantitativos señalan que los hombres tienden a subestimar su peso, y aunque, como ya se mencionó, no se pueda establecer comparaciones por las diferencias metodológicas de los estudios, los datos derivados del trabajo realizado en San Martín Cachihuapan abren la puerta para continuar investigando este tema en comunidades rurales a través de un abordaje cualitativo y una perspectiva de género.

Se considera que otro tema pendiente es identificar y profundizar acerca de cómo los parámetros del cuerpo ideal de las mujeres y hombres que viven en situaciones de desventaja social y económica y, por supuesto, alimentaria son construidos por el grupo social y cultural inmediato, por ejemplo, la familia de origen, el sector salud y los medios masivos de comunicación. La familia es la trasmisora de patrones culturales y a través de ella se crean y recrean las maneras de concebir al cuerpo, desde la expresión de las emociones, el contacto corporal, el cuidado del 
cuerpo, la estética, la normalidad del cuerpo y de la alimentación y los valores asociados al cuerpo con todas su manifestaciones físicas y emocionales, expresiones de enfermedad, del cuidado y la alimentación. Cómo se transmiten esos patrones es un tema que debe investigarse en profundidad, así como el discurso biomédico que, en los últimos años, ha cambiado el estado epistemológico de la obesidad, que ha pasado de ser considerada un simple factor de riesgo a una patología crónica y, más recientemente, a ser una epidemia mundial..$^{17,18}$

La inclusión de la percepción corporal y el ideal del cuerpo en los programas de alimentación y nutrición son relevantes cuando se busca prevenir ciertos trastornos como el sobrepeso, la obesidad, la anorexia y la bulimia, y profundizar en los significados que tienen para las mujeres y los hombres el acto de comer o no comer determinados alimentos o productos "buenos para transformar el cuerpo". Abordar la imagen corporal desde una perspectiva de género con el propósito de identificar las similitudes o diferencias entre hombres y mujeres en tres de las áreas definidas como centrales en el uso del cuerpo, es decir, la alimentación, el ejercicio físico y el cuidado estético, es una temática imprescindible.

\section{Colaboradores}

Juárez AGR y Romo SEPG participaron en la concepción, análisis, interpretación y redacción de la versión final del texto. Rosa Cruz SA participó en la recolección de los datos y en el análisis de la información.

Conflicto de Intereses: Los autores declaran no haber conflicto de intereses.

\section{Referencias}

1. Instituto Nacional de Salud Pública. Encuesta Nacional de Salud y Nutrición 2012. Cuernavaca, México: Instituto Nacional de Salud Pública; 2012.

2. Instituto Nacional de Salud Pública. Encuesta Nacional de Salud 2000. Tomo 2. La salud de los adultos. Cuernavaca, México: Instituto Nacional de Salud Pública; 2003.

3. Instituto Nacional de Salud Pública. Encuesta Nacional de Salud y Nutrición 2006. Cuernavaca, México: Instituto Nacional de Salud Pública; 2006.

4. González de León D, Bertran M, Salinas AA, Torre P, Mora F, Pérez Gil SE. La epidemia de Obesidad y las mujeres. Revista Género y Salud en Cifras 2009; 7(1):17-29.

5. Arroyo P, Loria A, Fernández V, Flegal K, Kuri P, Olaiz G, et al. Prevalence of pre-obesity and obesity in urban adult Mexicans in comparison with other large surveys. Obesity Research 2000; 8(2):179-185. 
6. González C, Stern M. La obesidad como factor de riesgo cardiovascular en México: estudio en población abierta. Inv Clínica 1993; 45(1):13-21.

7. Instituto Nacional de la Nutrición Salvador Zubirán. Encuesta urbana de alimentación y nutrición en la zona metropolitana de la Ciudad de México 1995. México: Instituto Nacional de Nutrición Salvador Zubirán; 1995.

8. Ávila A, Shamah T, Chávez A, Galindo C. Encuesta urbana de alimentación y nutrición en la zona metropolitana de la Ciudad de México 2002. México: Instituto Nacional de Nutrición Salvador Zubirán; 2003.

9. Unikel C, Bojórquez I, Carreño S, Validación de un cuestionario bree para medir prácticas alimentarias de riesgo. Rev Salud Publica 2004; 46(6):509-515.

10. Uribe Merino JF. Las prácticas alimentarias relacionadas con la búsqueda del ideal corporal: el caso de la ciudad de Medellín, Colombia. Boletín de Antropología Universidad de Antioquia 2006; 20(37):227-250.

11. Unikel C, Bojórquez I, Villatoro J, Fleiz C, Medina-Mora ME. Conductas alimentarias de riesgo en población estudiantil: datos en población estudiantil del Distrito Federal. Inv Clínica 2006; 58:(1):15-27.

12. Unikel C, Villatoro J, Medina-Mora ME, Fleiz BC, Alcántar ME. Conductas alimentarias de riesgo en adolescentes mexicanos. Datos en población estudiantil del Distrito Federal. Salud Mental 2000; 52(2):140-147.

13. Unikel C, Díaz de León C, Gonzalez C, Wagner F, Rivera JA. Conducta alimentaria de riesgo, síntomas depresivos y correlatos psicosociales en estudiantes universitarios de primer ingreso. Acta Universitaria 2015; 25(2):35-39.

14. Gracia Arnaiz M. Els trastorns alimentaris a Catalunya: una aproximació antropológica. Barcelona, España: Generalitat de Catalunya, Secretaria de Juventut; 2007. Collecció Estudis, n. 23.

15. Esteban ML. Antropología del cuerpo. Género, Itineraries corporals, identidad y cambio. Barcelona, España: Bellatierra; 2004.

16. Contreras J, Gracia Arnaiz M. Alimentación y cultura. Perspectivas antropológicas. Barcelona España: Ariel; 2005.

17. Gracia Arnaiz M. Maneras de comer hoy. Comprender la modernidad alimentaria, desde y más allá de las normas. Rev Int Sociol. 2005; 40:140-182

18. Gracia M. Paradojas de la alimentación contemporánea. Barcelona, España: Icaria; 2006.

19. Contreras J. Introducion. In: Contreras J, compilador. Alimentación y cultura: necesidades, gustos y costumbres. Barcelona, España: Universitat de Barcelona; 1995. p 9-26.

20. Toro J. El cuerpo como delito: anorexia, bulimia, cultura y sociedad. Barcelona, España: Ariel Ciencia; 1997.

21. Raich R. Una perspectiva desde la psicología de la salud de la imagen corporal. Avances en Psicología Latinoamericana 2004; 22:15-27.

22. Cash TF, Pruzinsky T, editores. Body images: a handbook of theory, research, and clinical practice. New York: Guilford Press; 2002. 
23. Connell RW. La organización social de la masculinidad. In: Valdés T, Olavarría O, editores. Masculinidades: poder y crisis. Chile: Isis Internacional; 1997. p. 31-48.

24. Toro-Alfonso J, Walters-Pacheco KZ, Sánchez Cardona I. El cuerpo en forma: masculinidad, imagen corporal y transtornos en la conducta alimentaria de atletas varones universitarios. Acta de Investigación Psicológica 2012; 2(3):842-857.

25. Pérez-Gil SE. Un problema actual: decidir entre comer y no comer. Cuadernos de Nutrición 2009; 32(6):205-212.

26. Schild P. The image and appearance of the human body. New York: Int Univ Press; 1950.

27. Garner DM. Inventario de trastornos de la conducta alimentaria (EDI 2). Madrid: Tea Ediciones; 1998.

28. Bergstrom RL, Neighbors C. Body image disturbance and the social norms approach: an integrative review of the literature. Journal of Social and Clinical Psychology 2006; 25(9):975-1000.

29. Fischler C. Gastro-nomía y gastro-anomía: sabiduría del cuerpo y crisis biocultural de la alimentación contemporánea. In: Contreras J, compilador. Alimentación y cultura: necesidades gustos y costumbres. Barcelona, España: Ediciones Universidad de Barcelona; 2002. p. 357-380.

30. Silberstein B, Perdue L, Peterson B, Kelly E. The role of the mass media in promoting a thin standard of bodily attractiveness for women. Sex Roles 1986; 14(9/10):519-532.

31. Gómez E. Equity, gender, and health: challenges for action. Pan Am J Public Health 2002; 11(5/6):454-561.

32. Salazar Z. Adolescencia e imagen corporal en la época de la delgadez. Reflexiones 2008; 87(2):67-80.

33. Saucedo Molina TJ, Unikel Santocini C. Conductas alimentarias de riesgo, interiorización del ideal estético de delgadez e índice de masa corporal en estudiantes hidalguenses de preparatoria y licenciatura de una institución privada. Salud Mental 2010; 33(1):11-19.

34. Pérez Gil SE, Vega A, Romero G. Alimentación de mujeres en una zona rural: ¿existe una nueva percepción del cuerpo?. Rev Salud Pública Mex. 2007; 49(1):52-62.

35. Gil SE, Romero G. Imagen corporal en mujeres rurales de la Sierra Juárez y la costa de Oaxaca: una aproximación nutrio-antropo-lógica. Revista de Estudios Sociales 2008; XVI(32):79-111.

36. Pérez O, Estrella D. Percepción de la imagen corporal y prácticas alimentarias entre indígenas mayas de Yucatán, México. Rev Chil Nut. 2015; 41(4):385-392.

37. Keijzer B, Rodriguez G. Jóvenes rurales. Género y generación en un mundo cambiante. In: Olavarría J, Editor. Varones adolescentes: genero, identidades: sexualidades en America Latina. Chile, UNFPA; 2003. p. 33-52.

38. Keijzer B. Hasta donde el cuerpo aguante: género, cuerpo y salud masculina. Revista la Manzana 2006; 1(1):137-152.

39. Núñez G. Los “hombres" en los estudios de género de los "hombres”: un reto de los estudios queer. In: Ramírez Rodríguez JC, Uribe Vázquez G, coordenadores. Masculinidades. El juego de género de los hombres en el que participan las mujeres. Guadalajara: Plaza y Valdés; 2008. 
40. Fuentes A. El discurso sobre la estética del cuerpo de los hombres. In: Ramírez JC, Uribe G. Masculinidades. El juego de género de los hombres en el que participan las mujeres. Guadalajara: Plaza y Valdés 2008.

41. Quapper D, Orlando C. O. Cuerpo poder y placer: disputas en hombres jóvenes de sectores empobrecidos. Revista de Psicología. Universidad Academia de Humanismo Cristiano 2005; 6(9):71-84.

42. SEDESOL. Catálogo de Localidades. Ciudad de México: 2016. Disponible en: http://www. microrregiones.gob.mx/catloc/contenido.aspx?refnac $=151120024$

43. Rueda-Jaime GE, Camacho PA, Flores M, Rangel AM. Validez y confiabilidad de dos escalas de siluetas para valorar la imagen corporal en estudiantes adolescentes. Rev Colomb Psiquiatr. 2012; 40(1):101-110.

44. Pérez Gil-SE, Díez Urdanivia S. El abordaje cualitativo: una alternativa en las investigaciones y acciones de alimentación comunitaria. Rev. Nutrición Clínica 2007; 10(1):28-35.

45. Garner RM, Stark K, Jackson NA, Friedman BN. Development and validation of two new scales for assessment of body image. Percept Mot Skills 1999; 89(3 Pt. 1):981-93.

Recibido: 25.4.2016

Revisado: 22.11.2016

Aceptado: 28.1.2017 